\title{
Immunogenicity and Safety of an Inactivated Hepatitis A Vaccine Given with Measles-Mumps-Rubella Vaccine to 12-13 Month Old Turkish Children
}

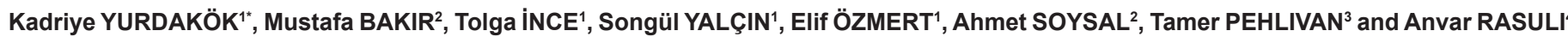

${ }^{1}$ Hacettepe University Faculty of Medicine, Department of Pediatrics, Social Pediatrics Unit, Ankara, Turkey

${ }^{2}$ Marmara University Hospital, Department of Pediatrics, Division of Pediatric Infectious Diseases, Istanbul, Turkey

${ }^{3}$ Sanofi Pasteur, Istanbul, Turkey

${ }^{4}$ Sanofi Pasteur, Lyon, France

\begin{abstract}
Background: The epidemiology of hepatitis A in Turkey favors routine vaccination of children. This study assessed compatibility of a monovalent hepatitis $A$ vaccine with the measles-mumps-rubella (MMR) combination vaccine used in the national immunization calendar at 12-13 months of age.

Methods: Hepatitis A seronegative participants were randomized equally to hepatitis $A$ vaccine followed by MMR vaccine 28 days later (Group A), MMR vaccine followed by hepatitis A vaccine 28 days later (Group B), or one dose each of hepatitis A and MMR vaccines given on Day 0 (Group C). All participants received a hepatitis A booster dose 6 months later.

Results: A total of 470 seronegative (anti-hepatitis A concentration $\geq 20 \mathrm{mlU} / \mathrm{mL}$ ) participants were randomized: 188 to Group A, 94 to Group B and 188 to Group C. The hepatitis A seroprotection rates ( $\geq 20 \mathrm{mlU} / \mathrm{mL}), 1$ month after the first vaccination were $93.6 \%$ (Group A) and $92.7 \%$ (Group C) by a microparticle enzyme immunoassay (MEIA). Using a more sensitive electrochemiluminescence immunoassay (ECLIA), the corresponding seroprotection rates were 100 and $99.4 \%$. Non-inferiority of seroprotection against hepatitis A was demonstrated for concomitant versus non-concomitant vaccination using ECLIA, but not MEIA. After booster, all participants had anti-hepatitis titers $\geq 20$ $\mathrm{mIU} / \mathrm{mL}$ and anti-hepatitis geometric mean concentrations of $5,078 \mathrm{mlU} / \mathrm{mL}$ (Group A), 3,271 mIU/mL (Group B) and $4,314 \mathrm{mlU} / \mathrm{mL}$ (Group C). Following primary vaccination, measles $(\geq 120 \mathrm{mlU} / \mathrm{mL})$ and mumps $(\geq 10 \mathrm{AU} / \mathrm{mL})$ seroprotection rates were $96.5 \%$ with both separate and concomitant vaccination. The rubella seroprotection $(\geq 10$ $\mathrm{IU} / \mathrm{mL}$ ) rates were 97.6 and $96.7 \%$ following separate and concomitant vaccination, respectively. Reactogenicity increased slightly with concomitant administration; both vaccines were well tolerated.
\end{abstract}

Conclusions: The immune response to the hepatitis A vaccine was not impaired by concomitant administration with MMR vaccine. Non-inferiority of seroprotection was demonstrated with the more sensitive of two assays used to evaluate anti-hepatitis A antibody.

Keywords: Hepatitis A vaccine; Co-administration; Pediatric vaccines; Measles, Mumps rubella vaccine

\section{Introduction}

In 2005, following a successful vaccination campaign to reduce the incidence of measles in Turkey, the National Immunization Program (NIP) replaced monovalent measles vaccination at 9 months of age with combination measles, mumps, rubella (MMR) combination vaccine given at 12 months and at 6 years of age [1]

The epidemiology and burden of hepatitis A also favors routine universal vaccination of children in Turkey. The World Health organization (WHO) classifies hepatitis A virus (HAV) endemicity as high, intermediate or low, depending on the prevalence of anti-HAV (IgG) antibodies in sera of specific age groups [2]. Improved hygiene and sanitation result in an overall reduction in HAV circulation and decrease of endemicity from high to intermediate and from intermediate to low. Epidemiologic monitoring and accurate surveillance of vaccinepreventable diseases play an important role in the selection of vaccines for inclusion in the NIP and the vaccination strategies that are followed. Evidence of decreasing HAV seroprevalence in younger age groups for over more than a decade has been reported in Turkey [3-5]. People who are highly susceptible - because of decreased seroprevalence - may live together with people among whom the HAV widely circulates. Consequently, many individuals escape early childhood infection and are infected later in life when they are more likely to develop severe hepatitis A disease. A survey of HAV seroprevalence in Turkey demonstrated clear differences among five regions, with seropositivity in children between 5-9 years of age $>80 \%$ in the south-east and east, and $<50 \%$ in central and western regions [6]. Hepatitis $\mathrm{A}$ is a reportable disease in Turkey. Hepatitis A incidence in the Izmir region in 2003 was estimated at 27.66/100,000 from case and laboratory reports or 53 cases/100,000 by the capture-recapture method [7]. Seroprevalence and surveillance data in Turkey indicate intermediate endemicity, supporting routine childhood hepatitis A vaccination.

As with MMR, the first dose of hepatitis A vaccine is generally

*Corresponding author: Kadriye YURDAKÖK, Hacettepe University Faculty of Medicine, Department of Pediatrics, Social Pediatrics Unit, Ankara, Turkey, Tel: +90 31230511 33; Fax: +90 31232432 84; E-mail: yurdakok@bilkent.edu.tr

Received September 10, 2012; Accepted September 20, 2012; Published September 25, 2012

Citation: YURDAKÖK K, BAKIR M, INCE T, YALÇIN S, ÖZMERT E, et al. (2012) Immunogenicity and Safety of an Inactivated Hepatitis A Vaccine Given with Measles-Mumps-Rubella Vaccine to 12-13 Month Old Turkish Children. J Vaccines Vaccin 3:146. doi:10.4172/2157-7560.1000146

Copyright: ( 2012 YURDAKÖK K, et al. This is an open-access article distributed under the terms of the Creative Commons Attribution License, which permits unrestricted use, distribution, and reproduction in any medium, provided the original author and source are credited. 
given at 12 months of age. Giving both vaccines at the same time would avoid separate clinic visits and would potentially assure the new vaccine would have coverage as high as the existing MMR vaccine. Before adding hepatitis A vaccination to the NIP, the safety and immunogenicity of the vaccine given concomitantly with MMR vaccine at different injection sites must be documented. Previous studies have shown hepatitis A and MMR vaccines were compatible when given concomitantly, but the data is limited [8-10]. Therefore, this open, randomized, controlled study was conducted to assess whether hepatitis $A$ and MMR vaccination (Avaxim $^{\text {ma }} 80 \mathrm{U}$ Pediatric and Trimovax ${ }^{\mathrm{m}}$, Sanofi Pasteur, Lyon, France) could be administered concomitantly at 12 to 13 months of age to children.

\section{Material and Methods}

\section{Trial design and participants}

This Phase IV, observer-blind trial (Clinicaltrials.gov NCT00313950) was conducted at two centers in Turkey. The Ethics Committee/Institutional Review Board at each study center approved the protocol, written informed consent forms, recruitment procedures and other written information provided to participants. The trial was conducted in accordance with the Helsinki Declaration, International Conference on Harmonization and Good Clinical Practice guidelines. The participant's parent(s)/legal representative gave written informed consent before enrolment.

Healthy children, 12-13 months of age, born at full term pregnancy (>37 weeks) with a birth weight of $\geq 2.5 \mathrm{~kg}$, were eligible if they were hepatitis A seronegative. Receipt of one dose of monovalent measles vaccine at 9 months of age and before enrollment was allowed. Participants were excluded if they were hepatitis A seropositive, were enrolled in another clinical trial during the 4 weeks prior to first study vaccination, or planned to participate in another clinical trial during the course of this trial; had congenital or acquired immunodeficiency; received immunosuppressive therapy such as long-term systemic corticosteroids therapy; were allergic to any study vaccine component; had a chronic illness that could interfere with trial conduct or completion; had received blood or blood-derived products in the past 3 months; had any vaccination in the past 4 weeks, or planned in the 4 weeks following the first trial vaccination; history of (confirmed) hepatitis A, measles, mumps, and/or rubella infection; previous hepatitis A or MMR vaccination; thrombocytopenia or a bleeding disorder contraindicating intramuscular (IM) injections; history of seizures; or a febrile illness (axillary temperature $\geq 37.4^{\circ} \mathrm{C}$ ) on the day of enrolment.

Participants were screened for hepatitis A seropositivity after they gave informed consent. Seronegative participants were randomly assigned equally to one of three study groups: Hepatitis A vaccine followed by MMR 28 days later (Group A), MMR vaccine followed by hepatitis A vaccine 28 days later (Group B), or one dose of hepatitis $A$ vaccine and one dose of MMR given at two different sites on Day 0 and Day 28 (Group C). All participants received a hepatitis A vaccine booster dose 6 months after the first dose. Each participant was followed-up for approximately 8 months.

Group assignment was performed using a list of random numbers supplied by the study sponsor. As both vaccines were commercially available and easily identifiable, the study design was observer-blind, with only the personnel who administered the vaccines being aware of the study group assignments. The investigators, parent(s)/guardian(s) did not witness administration.

\section{Vaccines}

Avaxim $^{\text {Tw }} 80 \mathrm{U}$ Pediatric is an inactivated hepatitis A vaccine containing 80 antigen units of formaldehyde-inactivated GBM strain hepatitis A virus in each $0.5 \mathrm{~mL}$ dose. Trimovax ${ }^{\mathrm{ma}}$ is a trivalent MMR vaccine containing $\geq 1,000$ tissue culture infective dose 50\% (TCID50) Schwarz strain measles, $\geq 5,000$ TCID50 Urabe Am9 strain mumps, and $\geq 1,000$ TCID50 Wistar RA 27/3M rubella live attenuated viruses in each $0.5 \mathrm{~mL}$ dose. Both vaccines are licensed in Turkey and were provided by Sanofi Pasteur, Lyon, France. The hepatitis A vaccine (commercial batches Y1063-1, A1182-2 and Z0532-1) was supplied as a liquid suspension in single-dose prefilled syringes. The MMR vaccine (batches Z5334-2, Z6638-5, A5715-1 and B5576-4) was supplied as a freeze-dried powder in single-dose vials and reconstituted with water for injection. Both vaccines were administered by IM injection into the deltoid muscle.

\section{Laboratory methods}

Four blood samples were collected: 7 days prior to enrolment in order to determine hepatitis A sero-status and for measurement of prevaccination antibody concentrations, at 4 weeks (Day 28), at 7 months after the first vaccination (Day 213), and 4 weeks after hepatitis A booster vaccination (Day 243).

Anti-Hepatitis A antibody assessment: Anti-hepatitis A antibody concentrations at enrollment, after primary vaccination, before and after booster vaccination were measured at BARC laboratories in Ghent, Belgium by using a commercially available microparticle enzyme immunoassay (MEIA) (Abbott Axsym HAVAB 2.0, Abbott Park, Illinois, USA). The lower limit of quantification (LLOQ) for that assay was $5 \mathrm{mIU} / \mathrm{mL}$. The cut off value for HAV seropositivity was defined as $20 \mathrm{mIU} / \mathrm{mL}$; participants with concentrations $<20 \mathrm{mIU} / \mathrm{mL}$ at screening were considered seronegative and eligible for the trial. As no absolute threshold of hepatitis A seroprotection has been identified [11], both $5 \mathrm{mIU} / \mathrm{mL}$ and $20 \mathrm{mIU} / \mathrm{mL}$ were used in the immunogenicity analysis.

Additional serological testing was performed at the same laboratory using a commercially available electrochemiluminescence immunoassay (ECLIA) (Roche Elecsys Anti-HAV, Roche Diagnostics, Indianapolis, USA) to measure hepatitis A IgM and IgG in postscreening sera from the enrolled subjects, including post first-dose sera from Groups A and C on Day 28, and pre-booster samples from all three study groups. The LLOQ for the assay was $3 \mathrm{mIU} / \mathrm{mL}$. The ECLIA test results were analyzed following the statistical plan for Group A and Group C seroprotection rates after the first vaccine dose and prior to administration of the booster dose. With the ECLIA test, both $\geq 5 \mathrm{mIU} /$ $\mathrm{mL}$ and $\geq 20 \mathrm{mIU} / \mathrm{mL}$ thresholds were considered for the hepatitis A seroprotection rate.

Anti-measles, mumps and rubella antibody assessment: AntiMeasles, mumps and rubella antibody concentrations at enrollment and 1 month after vaccination were measured by enzyme-linked immunosorbent assay (ELISA) at PPD Laboratories in Wayne, PA, USA. The LLOQs for the assays were $60 \mathrm{mIU} / \mathrm{mL}$ for measles, 5 antigen units (AU) per $\mathrm{mL}$ for mumps and $5 \mathrm{IU} / \mathrm{mL}$ for rubella. The cut off values for seropositivity/seroprotection were defined as $120 \mathrm{mIU} / \mathrm{mL}$ for measles, $10 \mathrm{IU} / \mathrm{mL}$ for rubella, and $10 \mathrm{AU} / \mathrm{mLfor}$ mumps.

\section{Safety}

Subjects were monitored for immediate reactions in the first 30 minutes following each vaccine injection. Parents/guardians recorded 
Citation: YURDAKÖK K, BAKIR M, INCE T, YALÇIN S, ÖZMERT E, et al. (2012) Immunogenicity and Safety of an Inactivated Hepatitis A Vaccine Given with Measles-Mumps-Rubella Vaccine to 12-13 Month Old Turkish Children. J Vaccines Vaccin 3:146. doi:10.4172/2157-7560.1000146

Page 3 of 7

the occurrence, intensity and duration of solicited injection site (tenderness, redness, swelling) and systemic (fever, vomiting, abnormal crying, drowsiness, appetite lost, irritability) reactions to occur on the day of vaccination and for 7 days afterwards. Unsolicited and serious adverse events (SAEs) were recorded during the whole study period. Information was collected in the source document and in the CRF for each subject.

\section{Statistical methods}

Seroprotection rates (SP) against hepatitis A at 4 weeks (Day 28) were assessed for non-inferiority of the concomitant administration of HAV and MMR vaccines (Group C) compared with administration of HAV and MMR on different days (Group A) using the 95\% confidence intervals (CI) of the SP difference and a clinically acceptable noninferiority limit of 5\%. To demonstrate non-inferiority, the $95 \%$ CI lower limit for the difference in SP rates between concomitant and separate vaccination had to be greater than $-5 \%$.

The sample size calculation indicated 170 participants would provide a power of $90 \%$ to demonstrate non-inferiority assuming a hepatitis A seroprotection $(\geq 20 \mathrm{mIU} / \mathrm{mL})$ rate of $99 \%$ and a clinically acceptable non-inferiority limit of 5\%. We decided to enroll 190 subjects in Groups A and C assuming a dropout rate of 10\%. A sample size of 90 participants was considered adequate for descriptive evaluation of immunogenicity in Group B.

In each study group, geometric mean concentrations (GMCs) with corresponding 95\% CIs and the seropositivity/seroprotection rates with $95 \%$ CIs for each of the vaccine antigens were calculated. Post- to pre-booster GMC ratios (GMCRs, geometric means of individual antiHAV concentration ratios) were also calculated with their 95\% CIs). All immunogenicity analyses were performed according to per-protocol and with the intent-to-treat (ITT) populations. Descriptive statistics were used for analysis of safety data.

\section{Results}

\section{Subject disposition}

A total of 654 participants were screened between 28 September 2006 and 06 February 2009. Of these, 470 were HAV seronegative (antiHAV concentration $\geq 20 \mathrm{mIU} / \mathrm{mL}$ ) and were randomized to three study groups: 188 to Group A (hepatitis A vaccine on Day $0+$ MMR on Day 28): 94 to Group B (MMR on Day 0 + hepatitis A vaccine on Day 28) and 188 to Group C (hepatitis A vaccine and MMR on Day 0 ). The three study groups were similar to each other with respect to gender and age of participants at enrollment; 55.3 to $57.4 \%$ of subjects were male and mean age was12.3 months (range, 11.6-13.3 months).

Of the 470 randomized participants, 436 (92.8\%) completed the primary phase and 398 (91.3\%) completed the booster phase. Figure 1 summarizes the disposition of participants. Two participants had an $\mathrm{AE}$ that led to premature discontinuation. One had acute gastroenteritis, which was judged unrelated to vaccination; the second had a grade 3 fever following vaccination and was withdrawn by the parents. The relation of fever to vaccination was not determined. No early withdrawal resulted from a SAE considered to be related to vaccine administration.

All vaccinated participants were included in the safety evaluation. The intent to treat analysis set for evaluation of primary vaccination at Day 28 consisted of all vaccinated participants with an evaluable blood sample. The per-protocol set consisted of $91.5 \%$ of participants in Group A, 90.4\% in Group B and 87.2\% in Group C. The most common reason for exclusion from the per-protocol analysis was absence of blood drawn on Day 28 followed by a too long interval between vaccination and blood sample collection exceeding the permissible 25 60 days. The post-booster immunogenicity analysis set included $81.9 \%$ of the participants in Group A, 85.1\% in Group B, and 78.7\% in Group

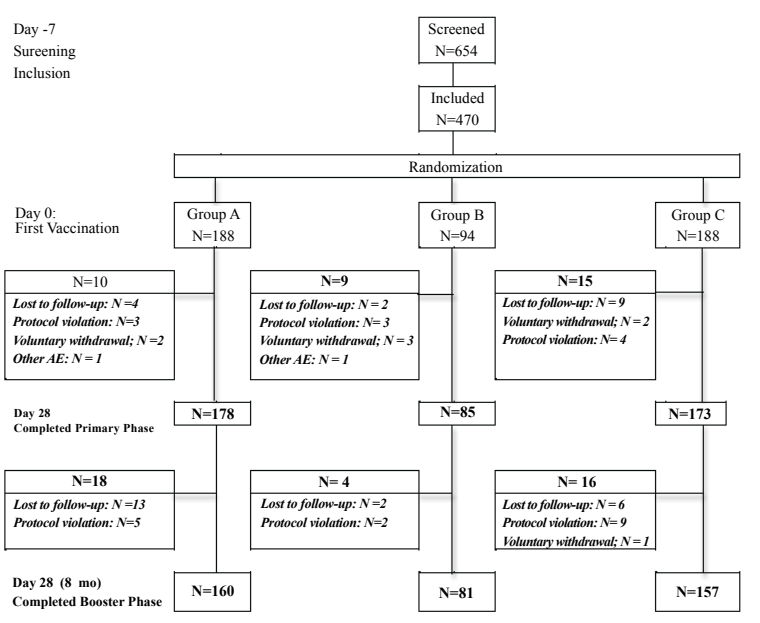

Figure 1: Participant disposition.

\begin{tabular}{|c|c|c|c|c|c|c|c|c|c|c|c|c|}
\hline & \multicolumn{4}{|c|}{ Group A: HAV vaccine Day 7 \& MMR vaccine Day 28} & \multicolumn{4}{|c|}{ Group B: MMR vaccine Day 7 \& HAV vaccine Day 28} & \multicolumn{4}{|c|}{ Group C: HAV vaccine \& MMR vaccine Day 0} \\
\hline & Day -7 & Day 28 & Day 213 & Day 243 & Day -7 & Day 28 & Day 213 & Day 243 & Day -7 & Day 28 & Day 213 & Day 243 \\
\hline N Data† & 172 & 172 & 154 & 154 & 85 & 84 & 79 & 80 & 164 & 164 & 148 & 148 \\
\hline $\begin{array}{l}\mathrm{GMC} \\
(\mathrm{mlU} / \mathrm{mL})\end{array}$ & 5.01 & 46.78 & 217.11 & 5078.74 & 5.06 & 3.67 & 97.92 & 3271.00 & 5.40 & 42.42 & 144.06 & 4314.86 \\
\hline$[95 \% \mathrm{Cl}]$ & {$[4.54 ; 5.53]$} & {$[42.43 ; 51.58]$} & {$[191 ; 246]$} & [4433;5818] & {$[4.38 ; 5.85]$} & [3.28;4.11] & {$[78.58 ; 122.02]$} & [2657.9;4025.6] & {$[4.83 ; 6.04]$} & [38.86;46.30] & {$[127.0 ; 163.4]$} & [3757.76;4954.54] \\
\hline $\begin{array}{l}\geq 5 \mathrm{mlU} / \mathrm{mL} \\
\% \text { (n) }\end{array}$ & $59.3(102)$ & $98.3(169)$ & $100.0(154)$ & $100.0(154)$ & $60.0(51)$ & $39.3(33)$ & $100.0(79)$ & $100.0(80)$ & $61.6(101)$ & $100.0(164)$ & $100.0(148)$ & $100.0(148)$ \\
\hline$[95 \% \mathrm{Cl}]$ & {$[50.87 ; 65.44]$} & [94.99;99.64] & {$[97.6 ; 100.0]$} & {$[97.63 ; 100.0]$} & {$[48.80 ; 70.48]$} & [28.80;50.55] & {$[95.44 ; 100.0]$} & [95.49;100.0] & [53.68;69.06] & {$[97.78 ; 100.0]$} & {$[97.54 ; 100.0]$} & {$[97.54 ; 100.0]$} \\
\hline $\begin{array}{l}\geq 20 \mathrm{mlU} / \\
\mathrm{mL} \% \text { (n) }\end{array}$ & $1.2(2)$ & $93.6(161)$ & $100.0(154)$ & $100.0(154)$ & $1.2(1)$ & $1.2(1)$ & $94.9(75)$ & $100.0(80)$ & $3.6(6)$ & $92.7(152)$ & $99.3(147)$ & $100.0(148)$ \\
\hline$[95 \% \mathrm{Cl}]$ & {$[0.14 ; 4.14]$} & {$[89.2 ; 96.8]$} & {$[97.6 ; 100.0]$} & {$[97.6 ; 100.0]$} & {$[0.03 ; 6.39]$} & {$[0.03 ; 6.46]$} & [87.54;98.60] & [95.49;100.0] & [1.35;7.79] & {$[87.57 ; 96.1]$} & [96.3;99.9] & [97.54;100.0] \\
\hline GMCR & NA & 9.34 & NA & 23.39 & NA & NA & NA & 33.50 & NA & 7.85 & NA & 29.95 \\
\hline$[95 \% \mathrm{Cl}]$ & & {$[8.07 ; 10.79]$} & & {$[20.7 ; 26.4]$} & & & & [28.36;39.58] & & {$[6.84 ; 9.01]$} & & {$[26.35 ; 34.05]$} \\
\hline
\end{tabular}

NA - Not Applicable; GMC - Geometric Mean Concentration; GMCR - Geometric Mean Concentration Ratio: geometric mean of individual anti-HAV concentration ratios, Day 28: Day 7 and Day 243: Day 213

Table 1: Anti-hepatitis A GMC, GMCR and seroprotection rates by micro enzyme immunosorbent assay (MEIA) at enrollment, one month after primary vaccination (Day 28), at booster administration (Day 213) and 1 month after booster administration (Day 243). 
Citation: YURDAKÖK K, BAKIR M, INCE T, YALÇIN S, ÖZMERT E, et al. (2012) Immunogenicity and Safety of an Inactivated Hepatitis A Vaccine Given with Measles-Mumps-Rubella Vaccine to 12-13 Month Old Turkish Children. J Vaccines Vaccin 3:146. doi:10.4172/2157-7560.1000146

Page 4 of 7

\begin{tabular}{|c|c|c|c|c|c|c|}
\hline & Day -7 & Day 28 & Day -7 & Day 28 & Day -7 & Day 28 \\
\hline $\mathrm{N}$ & 186 & 178 & 92 & 85 & 186 & 173 \\
\hline \multicolumn{7}{|l|}{ Measles $\mathrm{mIU} / \mathrm{mL}$} \\
\hline GMT & & 221.12 & & 2074.24 & & 2103.77 \\
\hline$[95 \% \mathrm{Cl}]$ & & {$[1.29 ; 37928.87]$} & & {$[1745.06 ; 2465.52]$} & & [1856.55;2383.90] \\
\hline$\geq 120 \mathrm{mlU} / \mathrm{mL}: \%$ (n) & $2.7(5)$ & $1.1(2)$ & $1.1(1)$ & $96.5(82)$ & $2.7(5)$ & $96.5(167)$ \\
\hline$[95 \% \mathrm{Cl}]$ & {$[0.88 ; 6.16]$} & {$[0.14 ; 4.00]$} & {$[0.03 ; 5.91]$} & {$[90.03 ; 99.27]$} & {$[0.88 ; 6.16]$} & {$[92.60 ; 98.72]$} \\
\hline \multicolumn{7}{|l|}{ Mumps (AU/mL) } \\
\hline GMT & & 24.91 & & 61.30 & & 64.92 \\
\hline$[95 \% \mathrm{Cl}]$ & & {$[4.58 ; 135.45]$} & & {$[52.48 ; 71.61]$} & & {$[58.52 ; 72.03]$} \\
\hline$\geq 10 \mathrm{AU} / \mathrm{mL}: \%$ (n) & $4.8(9)$ & $2.2(4)$ & $7.6(7)$ & $96.5(82)$ & $4.3(8)$ & $96.5(167)$ \\
\hline$[95 \% \mathrm{Cl}]$ & {$[2.24 ; 8.99]$} & {$[0.62 ; 5.65]$} & {$[3.11 ; 15.05]$} & {$[90.03 ; 99.27]$} & {$[1.87 ; 8.30]$} & [92.60;98.72] \\
\hline \multicolumn{7}{|l|}{ Rubella (IU/mL) } \\
\hline GMT & & 44.40 & & 92.85 & & 96.42 \\
\hline$[95 \% \mathrm{Cl}]$ & & & & {$[75.75 ; 113.82]$} & & [83.91;110.79] \\
\hline$\geq 10 \mathrm{IU} / \mathrm{mL}: \quad \%(\mathrm{n})$ & $0.5(1)$ & $0.6(1)$ & $0.0(0)$ & $97.6(83)$ & $0.5(1)$ & $96.5(167)$ \\
\hline$[95 \% \mathrm{Cl}]$ & {$[0.01 ; 2.96]$} & {$[0.01 ; 3.09]$} & {$[0.00 ; 3.93]$} & [91.76;99.71] & {$[0.01 ; 2.96]$} & {$[92.60 ; 98.72]$} \\
\hline
\end{tabular}

Table 2: Anti-measles, anti-mumps and anti-rubella GMC and seroprotection rates at enrollment and 1 month (Day 28) after vaccination.

\begin{tabular}{|c|c|c|c|c|c|}
\hline & \multicolumn{2}{|c|}{ Group $A^{*}$} & \multicolumn{2}{|c|}{ Group C* } & \multirow{2}{*}{$\begin{array}{l}\text { Group C - Group A } \\
\text { Difference }[95 \% \mathrm{Cl}]\end{array}$} \\
\hline & $\mathrm{n} / \mathrm{N}$ & $\%[95 \% \mathrm{Cl}\}$ & $n / N$ & $\%[95 \% \mathrm{Cl}\}$ & \\
\hline Anti-HAV ${ }^{1} \geq 20 \mathrm{mlU} / \mathrm{mL}$ & $161 / 172$ & $93.60[88.85 ; 96.76]$ & $152 / 164$ & $92.68[87.57 ; 96.16]$ & $-0.92[-6.68 ; 4.69]$ \\
\hline Anti-HAV ${ }^{1} \geq 5 \mathrm{mlU} / \mathrm{mL}$ & $169 / 172$ & 98.3 [94.99; 99.64] & $164 / 164$ & $100[97.78 ; 100.00]$ & $1.74[00.82 ; 5]^{*}$ \\
\hline Anti-HAV ${ }^{2} \geq 20 \mathrm{mlU} / \mathrm{mL}$ & $169 / 169$ & $100.0[97.7 ; 100.0]$ & $162 / 163$ & 99.39 [96.63; 99.98]; & $-0.61[-3.39 ; 1.67]^{\star}$ \\
\hline Anti-HAV ${ }^{2} \geq 5 \mathrm{mlU} / \mathrm{mL}$ & $172 / 172$ & $100[97.84 ; 100.0]$ & $163 / 164$ & $100[97.76 ; 100]$ & $-0.58[-3.33 ; 1.62]^{*}$ \\
\hline
\end{tabular}

Group A: HAV vaccine on Day 0 and MMR vaccine on Day 28

Group C: Concomitant HAV and MMR vaccines on Day 0

${ }^{*}$ Non-inferiority of Group C to Group A is demonstrated if the lower limit of the $95 \% \mathrm{Cl}$ of the difference is greater than $-5 \%$.

${ }^{1}$ MEIA Assay

${ }^{2}$ ECLIA Assay

Table 3: Anti-HAV Seroprotection Rate one month after primary vaccination (Day 28).

C. The most common reasons for exclusion were absence of booster vaccination and absence of a post-booster blood sample.

\section{Immunogenicity}

Hepatitis A: The seroprotection rates and GMCs for both hepatitis $A$ and MMR vaccines are summarized in Tables 1 and 2 . The hepatitis A seroprotection rates $(\geq 20 \mathrm{mIU} / \mathrm{mL}$, MEIA assay) one month after the first vaccination were $93.6 \%$ (161/172 participants) and $92.7 \%$ (152/164 participants) in Groups A and C, respectively. The difference in SP rates for Group C vs. Group A was -0.92\% (95\% CI: [-6.68; 4.69]). As the lower limit of the $95 \%$ CI was $<5 \%$, seroprotection following concomitant administration of the hepatitis $\mathrm{A}$ and MMR vaccines was not non-inferior to hepatitis A vaccine administered alone. Noninferiority of seroprotection $(\geq 20 \mathrm{mIU} / \mathrm{mL})$ against hepatitis A was demonstrated using the more sensitive ECLIA assay, which resulted in SP rates of $100 \%$ and $99.39 \%$ for non-concomitant and concomitant administration, respectively (Table 3 ). With a seroprotection threshold of $\geq 5 \mathrm{mIU} / \mathrm{mL}$, non-inferiority of concomitant vaccination was demonstrated by both the MEIA and ECLIA assays.

Before primary vaccination (Day 7), more than half of the participants had anti-HAV with concentrations $\geq 5 \mathrm{mIU} / \mathrm{mL}$. After a month this percentage decreased from 59.6 to $39.3 \%$ and the GMC decreased from 5.12 to $3.67 \mathrm{mIU} / \mathrm{mL}$ in Group B between enrollment and hepatitis A vaccination, suggesting that the anti-HAV antibodies present before vaccination were most likely of maternal origin.

Anti-HAV GMCs (MEIA assay) were low in all groups before primary vaccination, but reached $46.52 \mathrm{mIU} / \mathrm{mL}$ in Group A and 43.15
$\mathrm{mIU} / \mathrm{mL}$ in Group C 1 month following primary vaccination; post- to pre-booster GMCRs were 9.43 in Group A and 7.99 in Group C. At 6 months after primary vaccination (Day 213) all participants had antiHAV concentrations $\geq 5 \mathrm{mIU} / \mathrm{mL} ; 100 \%$ of those in Group A, $94.9 \%$ in Group B, and $99.3 \%$ in Group C had anti-HAV titers $\geq 20 \mathrm{mIU} / \mathrm{mL}$. GMCs were higher at 6 months than GMCs at 1 month after priming in all groups, ranging from $97.92 \mathrm{mIU} / \mathrm{mL}$ (Group B) to $217.11 \mathrm{mIU} /$ $\mathrm{mL}$ (Group A). Anti-HAV concentrations increased significantly in all groups at 1 month after booster administration (Day 243). All participants had anti-HAV titers $\geq 20 \mathrm{mIU} / \mathrm{mL}$. GMCs ranged from 3,271.00 $\mathrm{mIU} / \mathrm{mL}$ (Group B) to $5,078.74 \mathrm{mIU} / \mathrm{mL}$ (Group A). Postto pre-booster GMCRs were 23.39 in Group A, 33.50 in Group B, and 29.95 in Group C.

Anti-HAV antibody titers were higher at each time point when measured by ECLIA compared to MEIA. One month after primary vaccination (Day 28), GMCs were $538.41 \mathrm{mIU} / \mathrm{mL}$ and $339.54 \mathrm{mIU} / \mathrm{mL}$ in Group A and Group C respectively. In contrast to results obtained by the MEIA assay, GMTs measured by ECLIA decreased between primary and booster vaccination, to 315.83 and $205.19 \mathrm{mIU} / \mathrm{mL}$ in groups A and C, respectively. Before the booster dose (Day 213), 100\% of Group A, 94.9\% of Group B, and 99.3\% of group C participants had anti-HAV concentrations $\geq 20 \mathrm{mIU} / \mathrm{mL}$, and $100 \%$ of participants had concentrations $\geq 5 \mathrm{mIU} / \mathrm{mL}$.

Measles, mumps, and rubella: At enrollment, seroprotection rates (Table 2) were low, from $1.1 \%$ to $2.7 \%$ for measles $(\geq 120 \mathrm{mIU} / \mathrm{mL})$, $4.3 \%$ to $7.6 \%$ for mumps ( $\geq 10 \mathrm{AU} / \mathrm{mL}$ ) and $0.0 \%$ to $0.5 \%$ for rubella $(\geq 10 \mathrm{IU} / \mathrm{mL})$. None of the participants in the per protocol analysis 
Citation: YURDAKÖK K, BAKIR M, INCE T, YALÇIN S, ÖZMERT E, et al. (2012) Immunogenicity and Safety of an Inactivated Hepatitis A Vaccine Given with Measles-Mumps-Rubella Vaccine to 12-13 Month Old Turkish Children. J Vaccines Vaccin 3:146. doi:10.4172/2157-7560.1000146

Page 5 of 7

\begin{tabular}{|c|c|c|c|c|c|c|c|c|c|c|}
\hline & \multirow[b]{2}{*}{ Severity } & \multicolumn{3}{|c|}{ Group $A^{*}(N=188)$} & \multicolumn{3}{|c|}{ Group $B^{*}(N=94)$} & \multicolumn{3}{|c|}{ Group $C^{*}(N=188)$} \\
\hline & & $n / N$ & $\%$ & {$[95 \% \mathrm{Cl}]$} & $n / N$ & $\%$ & {$[95 \% \mathrm{Cl}]$} & $n / N$ & $\%$ & {$[95 \% \mathrm{Cl}]$} \\
\hline \multicolumn{11}{|c|}{ Injection site reactions } \\
\hline \multirow[t]{2}{*}{ Tenderness $\dagger$} & Any & $40 / 182$ & 22.0 & {$[16.2 ; 28.7]$} & $20 / 88$ & 22.7 & {$[14.5 ; 32.9]$} & $48 / 177$ & 27.1 & {$[20.7 ; 34.3]$} \\
\hline & Grade 3 & $2 / 182$ & 1.1 & {$[0.1 ; 3.9]$} & $0 / 88$ & 0.0 & {$[0.0 ; 4.1]$} & $4 / 177$ & 2.3 & {$[0.6 ; 5.7]$} \\
\hline \multirow[t]{2}{*}{ Erythema† } & Any & $14 / 182$ & 7.7 & {$[4.3 ; 12.6]$} & $7 / 88$ & 8.0 & {$[3.3 ; 15.7]$} & $26 / 177$ & 14.7 & {$[9.8 ; 20.8]$} \\
\hline & Grade 3 & 0/182 & 0.0 & {$[0.0 ; 2.0]$} & $0 / 88$ & 0.0 & {$[0.0 ; 4.1]$} & 0/177 & 0.0 & {$[0.0 ; 2.1]$} \\
\hline \multirow[t]{2}{*}{ Swelling $†$} & Any & 10/182 & 5.5 & {$[2.7 ; 9.9]$} & $5 / 88$ & 5.7 & {$[1.9 ; 12.8]$} & $15 / 177$ & 8.5 & {$[4.8 ; 13.6]$} \\
\hline & Grade 3 & $0 / 182$ & 0.0 & {$[0.0 ; 2.0]$} & $0 / 88$ & 0.0 & {$[0.0 ; 4.1]$} & 0/177 & 0.0 & {$[0.0 ; 2.1]$} \\
\hline \multicolumn{11}{|c|}{ Systemic reactions } \\
\hline \multirow[t]{2}{*}{ Fever } & Any & $11 / 182$ & 6.0 & {$[3.1 ; 10.6]$} & $8 / 88$ & 9.1 & {$[4.0 ; 17.1]$} & $22 / 177$ & 12.4 & {$[8.0 ; 18.2]$} \\
\hline & Grade 3 & $0 / 182$ & 0.0 & {$[0.0 ; 2.0]$} & $1 / 88$ & 1.1 & {$[0.0 ; 6.2]$} & $0 / 177$ & 0.0 & {$[0.0 ; 2.1]$} \\
\hline \multirow[t]{2}{*}{ Vomiting } & Any & $31 / 182$ & 17.0 & {$[11.9 ; 23.3]$} & $19 / 88$ & 21.6 & {$[13.5 ; 31.6]$} & $42 / 177$ & 23.7 & {$[17.7 ; 30.7]$} \\
\hline & Grade 3 & $1 / 182$ & 0.5 & {$[0.0 ; 3.0]$} & $1 / 88$ & 1.1 & {$[0.0 ; 6.2]$} & $2 / 177$ & 1.1 & {$[0.1 ; 4.0]$} \\
\hline \multirow[t]{2}{*}{ Abnormal crying } & Any & $47 / 182$ & 25.8 & {$[19.6 ; 32.8]$} & $30 / 88$ & 34.1 & {$[24.3 ; 45.0]$} & $58 / 177$ & 32.8 & {$[25.9 ; 40.2]$} \\
\hline & Grade 3 & $4 / 182$ & 2.2 & {$[0.6 ; 5.5]$} & $0 / 88$ & 0.0 & {$[0.0 ; 4.1]$} & $6 / 177$ & 3.4 & {$[1.3 ; 7.2]$} \\
\hline \multirow[t]{2}{*}{ Drowsiness } & Any & $24 / 182$ & 13.2 & {$[8.6 ; 19.0]$} & $14 / 88$ & 15.9 & {$[9.0 ; 25.2]$} & $36 / 177$ & 20.3 & {$[14.7 ; 27.0]$} \\
\hline & Grade 3 & $0 / 182$ & 0.0 & {$[0.0 ; 2.0]$} & $0 / 88$ & 0.0 & {$[0.0 ; 4.1]$} & $0 / 177$ & 0.0 & {$[0.0 ; 2.1]$} \\
\hline \multirow[t]{2}{*}{ Appetite lost } & Any & $62 / 182$ & 34.1 & {$[27.2 ; 41.4]$} & $28 / 88$ & 31.8 & {$[22.3 ; 42.6]$} & $73 / 177$ & 41.2 & [33.9;48.9] \\
\hline & Grade 3 & $7 / 182$ & 3.8 & {$[1.6 ; 7.8]$} & 9/88 & 10.2 & {$[4.8 ; 18.5]$} & $12 / 177$ & 6.8 & {$[3.6 ; 11.5]$} \\
\hline \multirow[t]{2}{*}{ Irritability } & Any & $83 / 182$ & 45.6 & {$[38.2 ; 53.1]$} & $47 / 88$ & 53.4 & {$[42.5 ; 64.1]$} & $91 / 177$ & 51.4 & [43.8;59.0] \\
\hline & Grade 3 & 9/182 & 4.9 & {$[2.3 ; 9.2]$} & $7 / 88$ & 8.0 & {$[3.3 ; 15.7]$} & $14 / 177$ & 7.9 & {$[4.4 ; 12.9]$} \\
\hline
\end{tabular}

* Group A: HAV on Day 0 + MMR vaccine on Day 28; Group B: MMR vaccine on Day 0 + HAV on Day 28; Group C: HAV and MMR vaccine on Day 0. All groups received a booster dose of HAV on Day 213

$\dagger$ after at least one of the two vaccinations for Group C

Note: All solicited events are considered as reactions. For solicited reactions, the denominator for percentages is the number of vaccinated subjects for those at least one safety record for the concerned solicited reaction is available. Each subject is counted once and classified in the highest recorded severity score for the concerned solicited reactio

Grade 1, 2, and 3 pain were defined as 'minor reaction when injection site is touched,' 'cries and protests when injection site is touched,' and 'cries when injected limb is moved or the movement of the injected limb is reduced.' For erythema and swelling, a diameter of $<2.5 \mathrm{~cm}$ was assessed as Grade 1 , from 2.5 to $<5 \mathrm{~cm}$ as Grade 2 and $\geq 5 \mathrm{~cm}$ as Grade 3. Grade 1, 2, and 3 fever were defined as temperature $\geq 38.0^{\circ} \mathrm{C}-\leq 38.5^{\circ} \mathrm{C},>38.5^{\circ} \mathrm{C}-\leq 39.0^{\circ} \mathrm{C}$, and $>39.5^{\circ} \mathrm{C}$, respectively. Other systemic symptoms were defined as: vomiting (Grade 1-Grade 2, 1 to 5 episodes/day; Grade 3, $\geq 6$ episodes /day) abnormal crying (Grade 1-Grade 2, $\leq 3$ hours; Grade 3, >3 hours), drowsiness, (Grade 1-Grade 2, unusually sleepy; Grade 3, sleepy most of the time) loss of appetite (Grade 1-Grade 2, missed 1 to 2 meals; Grade 3 , missed $\geq 3$ meals) and irritability (Grade 1-Grade 2, easily consolable or needs increased attention; Grade 3, inconsolable)

Table 4: Summary of solicited reactions within 8 days after primary hepatitis A and MMR vaccination.

set had received monovalent measles vaccine before study enrolment. Following primary vaccination, measles and mumps seroprotection rates were $96.5 \%$ with both separate (Group B) and concomitant (Group C) vaccination. The rubella seroprotection rates were $97.6 \%$ and $96.7 \%$ following separate and concomitant vaccination, respectively. Similar, strong increases in anti-measles, -mumps and -rubella GMC were observed in response to vaccination with both regimens (Table 2).

\section{Safety}

Most solicited reactions after primary vaccination occurred in the first 4 days, lasted less than 3 days and were of grade 1 or grade 2 intensity. Approximately $62 \%$ of participants in Groups A and B and $71 \%$ in Group C reported solicited reactions following the first dose of hepatitis A or MMR vaccine (Table 4). The most frequently reported injection site reaction was tenderness, reported by $22-27 \%$ of participants in each group. In Groups A, B and C, occurrence of systemic reactions after the first dose of hepatitis A or MMR vaccine was $54.9 \%, 58.0 \%$, and $64 \%$ respectively. The most common systemic reactions were irritability, abnormal crying and loss of appetite. Only 2 participants (1.1\%) in Group A and 3 (1.7\%) in Group C reported Grade 3 injection site reactions after hepatitis A vaccination. Two participants in Group C reported grade 3 reactions after MMR vaccination. Grade 3 systemic solicited reactions were reported by 18 participants $(9.9 \%)$ in Group A, 14 (15.9\%) in Group B and 22 (11.9\%) in Group C.

Solicited reactions were slightly less frequent following the hepatitis A booster compared to primary vaccination. Following the HAV booster injection on Day 213, 44.0 to $46.9 \%$ of participants reported a solicited reaction; $22.5 \%$ to $26 \%$ percent experienced an injection site reaction, and $32.8 \%$ to $37.9 \%$ experienced a systemic reaction. From $2.2 \%$ to $4.5 \%$ reported Grade 3 injection site reactions and $5.7 \%$ to $9.6 \%$ reported grade 3 systemic reactions.

Overall, $0.5 \%$ of the subjects in Group A, $2.1 \%$ of the subjects in Group B, and $1.1 \%$ of the subjects from Group C reported at least one unsolicited reaction. One participant in Group A experienced a rash on the head and neck after hepatitis A vaccination. In Group $\mathrm{B}$, one participant experienced a systemic macro-papular rash and another experienced injection site bruising. One participant in Group $\mathrm{C}$ experienced injection site ecchymosis and another experienced a macro-papular rash. All were of Grade 1 intensity.

Unsolicited adverse events (AEs) were reported by $21.8 \%$ to $27.1 \%$ of each study group between enrollment and Day 28 . The vast majority were Grade 1 or Grade 2 systemic events that were childhood infections and considered to be unrelated to vaccine injection(s). Three Grade 3 unsolicited AEs were reported in 3 Group A participants, diagnosed as pneumonia and bronchiolitis requiring hospitalization in two participants and acute gastroenteritis in another, all were considered to be Systemic AEs (SAEs). A Grade 3 event, bronchiolitis in a Group $\mathrm{C}$ participant also required hospitalization and was considered as SAE. After the booster vaccination, $11.7 \%$ of the participants in Group A, 9.6\% in Group B, and 8.0\% in Group C experienced at least one unsolicited AE. All were of Grade 1 or Grade 2 intensity and all but one (a case of nasopharyngitis) were considered to be unrelated to vaccine 
injection. In all groups, the most common unsolicited systemic AEs were childhood infections.

Six participants experienced a total of 7 SAEs. Four were reported between enrollment and Day 28 ( 1 case of pneumonia and 1 case of bronchiolitis in Group A; 1 case of bronchopneumonia and 1 case of bronchiolitis in Group C). Three SAEs occurred between Day 28 and booster vaccination ( 1 case of cow milk allergy, and 2 cases of laryngitis). No SAE was considered by the investigator to be related to vaccine injections. None led to premature study discontinuation. No death was reported during the study period.

\section{Discussion}

The study results demonstrate that concomitant administration of the inactivated hepatitis A study vaccine did not interfere with the immunogenicity of the MMR vaccine currently used in the NIP of Turkey and did not diminish the seroprotective response to the hepatitis A vaccine. Descriptive comparisons of seroprotection rates one month after primary vaccination revealed no significant differences in the antibody responses elicited by concomitant versus non-concomitant administration of hepatitis A and MMR vaccines.

Although the hepatitis A post-primarySP rates $(\geq 20 \mathrm{mIU} / \mathrm{mL}$, MEIA assay) were high $(93.60 \%$ and $92.68 \%$, after separate and concomitant vaccination, respectively), they were lower than the expected $99 \%$ seen in previous study results [12]. Although the group difference in SP rates was $<1.0 \%$, non-inferiority of concomitant administration was not demonstrated, probably because the SP rates were lower than expected. Non-inferiority of seroprotection $(\geq 20 \mathrm{mIU} / \mathrm{mL})$ against hepatitis A was demonstrated using the more sensitive ECLIA assay, which resulted in SP rates of $100 \%$ and $99.39 \%$ for separate and concomitant administration, respectively.

The demonstration of a strong immune response to primary vaccination, i.e., a single dose of hepatitis $A$ vaccine is significant in light of the current public health interest in adopting one-dose hepatitis A vaccination in various National Immunization Programs [2]. There is evidence for the protective efficacy of a single dose of inactivated hepatitis vaccine from national surveillance in Argentina, where the study vaccine is used in the National Immunization Program, and from recent study reports [13-15]. A single dose may be a useful option for countries where the 2-dose schedule may not be possible, and only one dose can be administered universally or to special target groups. Additional studies of long-term immunogenicity and boosting potential are needed [2].

Few hepatitis A infections have been reported among vaccinated persons, and vaccine-induced antibody levels are generally high. Consequently, a hepatitis A seroprotection threshold following vaccination has not yet been identified. Anti-HAV concentrations of $20 \mathrm{mIU} / \mathrm{mL}$ after administration of immunoglobulins are known to protect against hepatitis A $[11,16]$. However, in vitro studies have shown that depending on the test system used, neutralizing antibodies against hepatitis A may still be present even if the measured concentration is less than $20 \mathrm{mIU} / \mathrm{mL}$ [17]. As no absolute protective level has been defined, the LLOQ of the assay used (i.e., detectable hepatitis A antibodies) is often considered to indicate seroprotection $[11,18]$. The LLOQ of the assay method used in this study was $5 \mathrm{mIU} / \mathrm{mL}$ for the MEIA and 3 $\mathrm{mIU} / \mathrm{mL}$ for the ECLIA assay; thus we have considered both $\geq 5$ and $\geq 20 \mathrm{mIU} / \mathrm{mL}$ as seroprotective.

Quantitation of anti-HAV concentration depends on the assay used, with varying sensitivity resulting from differences in design or reagents that affect the ability to detect low antibody concentrations $[17,19,20]$. As measured by the MEIA assay in this study, post primary anti-HAV antibody concentrations were lower than expected [12]. Nevertheless, our anti-HAV results are consistent with other reports that found the Elecsys Anti-HAV ECLIA assay to be more sensitive than the AxSYM HAVAB 2.0 MEIA assay [20,21]. In one series, the MEIA assay detected seroconversion in $10 \%$ fewer subjects 2 weeks after vaccination than the ECLIA assay, with part of the observed difference thought to have resulted from a differential ability to detect IgM [20]. Such a difference might account for both the relatively low anti-HAV concentrations observed by MEIA assay after primary vaccination in this study as well as increasing concentrations over the 6 months prior to booster administration.

From $58.3 \%$ to $62 \%$ of participants had anti-HAV concentrations above the lower limit of detection of the MEIA assay used for preenrollment screening. Those antibodies were most likely of maternal origin as the GMC decreased in Group B prior to administration of hepatitis A vaccine on Day 28. Seroepidemiological studies in Turkey have shown persistence of maternal anti-HAV for up to 18 months of age; seropositivity rates of infants at $3,6,9,12,15$, and 18 months of age were $90.5 \%, 84.4 \%, 62.6 \%, 36.1 \%, 13.6 \%$, and $6.1 \%$, respectively [22] The presence of maternal antibodies in our study participants did not interfere with priming and boosting with the study hepatitis A vaccine; a result that is consistent with previous reports [22-24]. Anti-HAV concentrations increased strongly in all study groups after the booster injection, suggesting that priming had been successful following both study schedules and regimens.

Seroprotection rates against measles, mumps and rubella and GMCs were low in all groups at enrollment and decreased in Group A until MMR vaccination on Day 28. The seroprotection rates of at least $96.5 \%$ in both Group B and Group C indicate that MMR vaccine induced a satisfactory immunological response when administered concomitantly with the hepatitis A vaccine.

The study vaccines were well tolerated, with solicited reactions generally of Grade 1 or 2 intensity and resolved without treatment within a few days. Reactions were less frequently reported after the booster dose than after the first dose. Reactogenicity was slightly greater following concomitant administration, with about $8 \%$ more participants reporting a solicited injection site reaction and $10 \%$ more reporting a solicited systemic reaction.

\section{Conclusion}

The immune response to the hepatitis A vaccine evaluated in this study, as measured by seroprotection rates and GMCs, was not impaired by concomitant administration with MMR vaccine. Noninferiority of concomitant, compared to separate administration of hepatitis A and MMR vaccines was demonstrated for seroprotection rates when measured with a sensitive assay for anti-hepatitis A antibody concentrations. While reactogenicity was slightly increased with concomitant administration of the two study vaccines, the vaccines were well tolerated.

\section{Acknowledgment}

The authors thank the children and their parents for participation, and also Serdar Altinel for his help for running the trial. The authors would also like to thank Clement Weinberger of The Stylus Medical Communication for assistance in the preparation of the manuscript on behalf of Sanofi Pasteur and in accordance with the European Medical Writers Association guidelines. 
Citation: YURDAKÖK K, BAKIR M, INCE T, YALÇIN S, ÖZMERT E, et al. (2012) Immunogenicity and Safety of an Inactivated Hepatitis A Vaccine Given with Measles-Mumps-Rubella Vaccine to 12-13 Month Old Turkish Children. J Vaccines Vaccin 3:146. doi:10.4172/2157-7560.1000146

\section{Disclosure}

TP and AR are employees of Sanofi Pasteur. KY, MB and AS have received honoraria from Sanofi Pasteur for conducting this clinical study. This study was conducted with the financial support of Sanofi Pasteur, Lyon, France.

\section{References}

1. Ceyhan M (2010) Recent improvements in the Turkish Childhood Nationa Immunization Program. Turk J Pediatr 52: 563-569.

2. World Health Organization (2012) WHO position paper on hepatitis A vaccines - June 2012. Wkly Epidemiol Rec 87: 261-276.

3. Erdoğan MS, Otkun M, Tatman-Otkun M, Akata F, Türe M (2004) The epidemiology of hepatitis a virus infection in children, in Edirne, Turkey. Eur J Epidemiol 19: 267-273.

4. 4. Kurugol Z, Aslan A, Turkoglu E, Koturoglu G (2011) Changing epidemiology of hepatitis A infection in Izmir, Turkey. Vaccine 29: 6259-6261.

5. Sac RU, Bostanci I, Dallar Y, Cihan G, Atli O (2009) Hepatitis A seroprevalence and demographics in Turkish children in Ankara. Pediatr Int 51: 5-8.

6. Ceyhan M, Yildirim I, Kurt N, Uysal G, Dikici B, et al. (2008) Differences in hepatitis A seroprevalence among geographical regions in Turkey: a need for regional vaccination recommendations. J Viral Hepat 15: 69-72.

7. Durusoy R, Karababa AO (2010) Completeness of hepatitis, brucellosis, syphilis, measles and HIVIAIDS surveillance in Izmir, Turkey. BMC Public Health 10: 71.

8. Dagan R, Amir J, Livni G, Greenberg D, Abu-Abed J, et al. (2007) Concomitant Administration of a Virosome-Adjuvanted Hepatitis A Vaccine With Routine Childhood Vaccines at Age Twelve to Fifteen Months: A Randomized Controlled Trial. Pediatr Infect Dis J 26: 787-793.

9. Usonis V, Meriste S, Bakasenas V, Lutsar I, Collard F, et al. (2005) Immunogenicity and safety of a combined hepatitis $A$ and $B$ vaccine administered concomitantly with either a measles-mumps-rubella or a diphtheria-tetanusacellular pertussis-inactivated poliomyelitis vaccine mixed with a Haemophilus influenzae type b conjugate vaccine in infants aged 12-18 months. Vaccine 23 : 2602-2606.

10. Rinderknecht S, Michaels MG, Blatter M, Gaglani M, Andrews W, et al (2011) Immunogenicity and safety of an inactivated hepatitis A vaccine when coadministered with measles-mumps-rubella and varicella vaccines in children less than 2 years of age. Pediatr Infect Dis J 30: e179-185.

11. Vidor E, Dumas R, Porteret V, Bailleux F, Veitch K (2004) Aventis Pasteur vaccines containing inactivated hepatitis A virus: a compilation of immunogenicity data. Eur J Clin Microbiol Infect Dis 23: 300-309.
12. Espul C, Benedetti L, Cuello H, Houillon G, Rasuli A (2012) Persistence of immunity from 1 year of age after one or two doses of hepatitis $A$ vaccine given to children in Argentina. Hepat Med 4: 53-60.

13. Mayorga Pérez O, Herzog C, Zellmeyer M, Loáisiga A, Frösner G, et al (2003) Efficacy of virosome hepatitis Avaccine in young children in Nicaragua: randomized placebo-controlled trial. J Infect Dis 188: 671-677.

14. Ministerio de Salud de la Nación. Boletín Epidemiológico Anual 2010. Available at: http://msal.gov.ar/htm/site/sala_situacion/PANELES/bep-anual-2010/ BEPANUAL_2010.pdf. Accessed August 6, 2012.

15. Hammitt, LL, Bulkow L, Hennessy TW, Zanis C, Snowball M, et al. (2008) Persistence of Antibody to Hepatitis A Virus 10 Years after Vaccination among Children and Adults. J Infect Dis 198: 1776-1782.

16. Fiore AE, Wasley A, Bell BP (2006) Prevention of hepatitis A through active or passive immunization: recommendations of the Advisory Committee on Immunization Practices (ACIP). MMWR Recomm Rep 55: 1-23.

17. Berger R, Just M, Althaus B (1993) Time course of hepatitis A antibody production after active, passive and active/passive immunisation: the results are highly dependent on the antibody test system used. J Virol Methods 43 287-297.

18. Van Damme P, Banatvala J, Fay O, Iwarson S, McMahon B, et al. (2003) Hepatitis A booster vaccination: is there a need? Lancet 362: 1065-1071.

19. Miller WJ, Clark W, Hurni W, Kuter B, Schofield T, et al. (1993) Sensitive assays for hepatitis A antibodies. J Med Virol 41: 201-204.

20. Wiedmann M, Boehm S, Schumacher W, Swysen C, Zauke M (2003) Evaluation of three commercial assays for the detection of hepatitis a virus. Eur J Clin Microbiol Infect Dis 22: 129-130.

21. Van Herck K, Crasta PD, Messier M, Hardt K, Van Damme P (2012) Seventeenyear antibody persistence in adults primed with two doses of an inactivated hepatitis A vaccine. Hum Vaccin Immunother.

22. Derya A, Necmi A, Emre A, Akgün Y (2005) Decline of maternal hepatitis A antibodies during the first 2 years of life in infants born in Turkey. Am J Trop Med Hyg 73: 457-459

23. Lagos R, MunozA, Dumas R, Pichon S, Zambrano B, et al. (2003) Immunological priming of one dose of inactivated hepatitis $A$ vaccine given during the first year of life in presence of maternal antibodies. Vaccine 21: 3730-3733.

24. López EL, Contrini MM, Xifró MC, Cattaneo MA, Zambrano B, et al. (2007) Hepatitis A vaccination of Argentinean infants: comparison of two vaccination schedules. Vaccine 25: 102-108. 